\title{
Adsorption kinetics in open nanopores as a source of low frequency noise
}

\author{
Simon Gravelle, ${ }^{\dagger}$ Roland R. Netz, ${ }^{*, \ddagger}$ and Lydéric Bocquet*, \\ $\dagger$ School of Engineering and Material Science, Queen Mary University of London, UK \\ $\ddagger$ Department of Physics, Freie Universität Berlin, 14195 Berlin, Germany \\ ฯ Laboratoire de Physique de l'Ecole Normale Supérieure, CNRS, Université PSL, \\ Sorbonne Université, Sorbonne Paris Cité, Paris, France \\ E-mail: rnetz@physik.fu-berlin.de; lyderic.bocquet@ens.fr
}

\begin{abstract}
Ionic current measurements through solid state nanopores consistently show a power spectral density that scales as $1 / f^{\alpha}$ at low frequency $f$, with an exponent $\alpha \sim 0.5-1.5$, but strikingly, the physical origin of this behavior remains elusive. Here we perform simulations of particles reversibly adsorbing at the surface of a nanopore, and show that the fluctuations in the number of adsorbed particles exhibit low-frequency pink noise. We furthermore propose theoretical modeling for the time-dependent adsorption of particles on the nanopore surface for various geometries, which predicts a frequency spectrum in very good agreement with the simulation results. Altogether, our results highlight that the low-frequency noise takes its origin in the reversible adsorption of ions at the pore surface combined with the long-lasting excursions of the ions in the reservoirs. The scaling regime of the power spectrum extends down to a cut-off frequency which is far smaller than simple diffusion estimates. Using realistic values for the pore dimensions and the adsorption-desorption kinetics, this predicts the observation of pink-noise for frequencies down to the hertz for a typical solid-state nanopore, in good agreement with experiments.
\end{abstract}

The fluctuations in the ionic current across a nanopore or nanochannel usually exhibit an anomalous low frequency dependence, in contrast to the expected white noise (flat) spectrum ${ }^{1}$. Experimentally, the noise spectrum is measured typically to scale as $S(f) \sim 1 / f^{\alpha}$ at low frequency $f$, with an exponent $\alpha \sim 0.5-1.5$. This behavior was reported in artificial nanopores and nanotubes with various shapes and materials ${ }^{2-11}$, but also in biological membranes ${ }^{12,13}$. This low-frequency noise does reduce the signal-to-noise ratio and impedes translocation measurement methods relying on the detection of the modulation of an electrical ionic current accompanying the passage of a macromolecule through a nanopore ${ }^{14-16}$. Such translocation methods are now applied for fast protein detection, genome mapping, or DNA sequencing ${ }^{17-25}$ and the low frequency noise reduces the sensitivity of these translocation measurements $^{3,26}$. Reversly, understanding the origin of this noise is of fundamental importance since it can be a precious source of information ${ }^{27}$, for example in electrochemical processes ${ }^{28-30}$, on the protonation of ionization sites $^{31}$, the dynamics of adsorption ${ }^{32}$, the transport of sugars $^{13,33-35}, \mathrm{ATP}^{36}$ and antibiotic molecules ${ }^{37}$, the impact of nanobubbles on the ionic current ${ }^{38}$, or as a probe for the measurement of mass flow ${ }^{39}$.

The origin of this low-frequency contribution in ionic current has remained elusive. Recent theoretical works have put forward the role of ion correlations ${ }^{41}$ as well as hydrodynamic interactions ${ }^{42}$, as a source of low frequency noise. On the experimental side, low frequency noise measured in carbon and boron-nitride nanotubes ${ }^{25}$ showed dependence on the surface charge. This suggests that surface effects and charge adsorption on the channel surface play an key role for the low-frequency spectrum. A usual result of the experiments is that the level of current noise obeys the so-called Hooge's low $S(f)=A \times\langle I\rangle^{2} / f^{\alpha}$, with $\langle I\rangle$ the mean current, and the coefficient $A$ being inversely proportional to the number of charge carriers in the sample ${ }^{6,25,43}$. This points to the fluctuations of the channel conductance, $G$, as the source of the noise spectrum. Indeed the current fluctuation $\delta I$ is related to the conductance fluctuations $\delta G$ according to $\left\langle\delta I^{2}\right\rangle(f)=\frac{\left\langle\delta G^{2}\right\rangle(f)}{\langle G\rangle^{2}} \times\langle I\rangle^{2}$, in line with Hooge's law ${ }^{10,25}$. Now, the ionic conductance is proportional to the number of charge carriers in the pore, so that its fluctuations originate in the fluctuations of the number of 'free' ions in the channel.

In this work we accordingly explore the role of adsorption-desorption kinetics of particles (charged and neutral) on the surface of nanopores: individual particles diffuse in the bulk of a nanopore, and possibly in the reservoirs, and experience adsorption-desorption processes on the nanopore surface. Using molecular simulations and theoretical modeling, we calculate the 
(A)

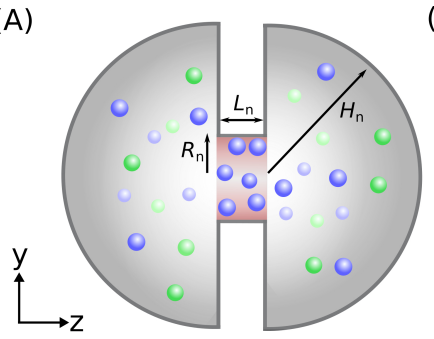

(B)

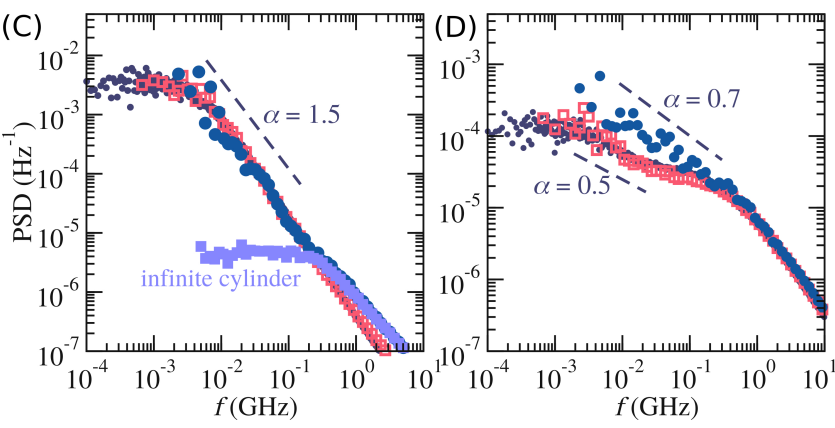

Figure 1: (A) Sketch of a cylindrical nanopore of length $L_{n}$, radius $R_{n}$, and connected to two half spherical reservoirs of radius $H_{n}$. The disks represent co-ions (green) and counterions (blue). (B) Snapshots of the molecular dynamics system ${ }^{40}$. The blue and green disks are the ions and the red disks are the traps. Solid flat walls are highlighted in gray. (C) PSDs of the number of bounded particles at the adsorbing surface as function of the frequency $f$ for a charged system (full disks), as well as for a neutral system (open squares), uncorrelated Brownian particles (black dots), and effectively infinite cylinder (full squares). The dashed line highlights the exponent as $f^{-\alpha}$ PSDs have been vertically shifted for clarity. (D) Corresponding PSDs of the number of free particles within the nanopore. The PSD for the infinite cylinder is not represented.

frequency spectrum of free and bounded particles in the nanopore. We explore how the geometry affects the low frequency spectrum, as well as the impact of the presence of reservoirs connected to the nanopore.

\section{Simulations.}

We start with the description of the process using Molecular Dynamics (MD) simulations. MD simulations are performed using LAMMPS ${ }^{44}$. A typical system is shown in Fig. $1 \mathrm{~A}$ and consists of a cylindrical nanopore (here with radius $R_{n}=2 \mathrm{~nm}$ and length $L_{n}=R_{n}$ ) connected to large half-spherical reservoirs (here with radius $H_{n}=20 \mathrm{~nm}$ ). The index ' $n$ ' stands for 'nanopore'. Charges are homogeneously distributed among the wall atoms at the surface with a surface charge $\Sigma$. A number $N=2 \pi R_{n} L_{n} \times \Sigma / e$ of monovalent counterions is initially placed in the nanopore in order to assure electroneutrality (here $N=5$ corresponding to $\Sigma \simeq 32 \mathrm{mC} / \mathrm{m}^{2}$ ); see Fig. 1 B. Monovalent ions of concentration $c_{s}=15 \mathrm{mM}$ are added to the reservoir. The temperature of the ions is fixed to $300 \mathrm{~K}$ thanks to a Langevin thermostat with a damping time $\tau=23 \mathrm{fs}^{45}$, leading to a diffusion coefficient $D \approx 1.5 \times 10^{-5} \mathrm{~cm}^{2} / \mathrm{s}$. The equations of motion are integrated using the velocity Verlet algorithm ${ }^{46}$, with a time-step equal to 2 fs. Long-range Coulombic in- teractions are calculated by means of a particle-particle particle-mesh (PPPM) solver ${ }^{47}$. In addition, particles interact through a Weeks-Chandler-Andersen potential $(\sigma=3 \AA)$, and the reversible adsorption of particles at the nanopore surface is introduced by adding a supplementary harmonic potential between fluid and wall atoms. A counter-ion forms a bond with a wall atom when the distance of their respective centers is lower than $\sigma$. The particle is then trapped to the surface site by a harmonic potential; the bond however breaks when the distance between the particle and the wall atom's center is higher than $\sigma$. The particle may thus escape thanks to thermal fluctuations. The depth of the harmonic potential, similar to the adsorption energy, is equal to $\epsilon=0.5 \mathrm{Kcal} / \mathrm{mol}$. This leads to an adsorption duration $T$ distributed as $P(T)=\lambda \exp (-\lambda T)$, with a desorption frequency $\lambda \approx 0.8 \mathrm{GHz}$. More details are given in the Appendix section. We record the number of bounded counter-ions $N_{B}$ at the cylinder surface as a function of time, as well as the total number of free (nonbounded) ions within the nanopore, $N_{F}$. Let us mention that the value for $\lambda$ in the simulations was chosen to be substantially larger than its experimental counterpart, typically in the range $\lambda_{\exp } \sim 10^{4} \mathrm{~Hz}^{31}$. This allows us to observe a sufficient number of desorption events within the range of the simulation time for proper statistics and quantify the influence of the adsorption-desorption kinetics in comparison to the theoretical predictions.

We calculate the Power Spectral Density (PSD) of both signals $N_{B}(t)$ and $N_{F}(t)^{48}$. As shown in Fig. $1 \mathrm{C}$, the PSD of the number of bounded particles $N_{B}$ scales as $1 / f^{\alpha}$ with $\alpha \approx 1.5$, for frequency $f$ in between $5 \cdot 10^{-3}$ and $5 \mathrm{GHz}$ (Fig. $1 \mathrm{C}$ ). Note that the fluctuations of $\delta N_{B}$ are proportional to the fluctuations of the surface charge density $\delta \Sigma$. Fig. $1 \mathrm{D}$ then shows the PSD of the number of free particles $N_{F}$ in the pore. The latter exhibits a frequency dependence behaving as $1 / f^{\alpha}$ with $\alpha \sim 0.7$ in between $5 \cdot 10^{-3}$ and $0.5 \mathrm{GHz}$, and $\alpha \sim 1.2$ for larger frequencies.

We compare these results with those obtained by simulations of three simpler model systems:

(i) We first considered a neutral system, for which the ions are replaced by a number $N=100$ of neutral particles and a charge-free surface. As shown in Fig. 1 C, (open symbols), this leads to almost identical PSD for the number of bounded particles. However, we find a substantial difference on the PSD of the number of free particles as compared to the charged case, since the scaling of the PSD for the neutral system exhibits a slightly lower exponent $(\alpha \sim 0.5)$ as compared to the charged system (Fig. 1D).

(ii) We also explored simulations of non-interacting particles, since correlations between ions were shown to be a potential source of pink noise ${ }^{41}$. We used a conventional Brownian dynamics algorithm with overdamped dynamics, implemented with a homemade C++ code (Method section). Strikingly, Brownian simulations 
lead to results very similar to neutral MD simulations (Fig. 1 CD), indicating that the observed pink noise is not a consequence of the correlations between the particles induced by the WCA potential. In addition, because Brownian simulations allow for the exploration of long duration at a low computational cost, the results reveal a plateau of the PSDs at frequencies below $f_{0}=4 \cdot 10^{-3} \mathrm{GHz}$, for both PSDs of the bounded $N_{B}(t)$ and free $N_{F}(t)$ particles. We will refer to $f_{0}$ as the low frequency cut-off.

(iii) Finally, we explored the PSD in an infinite cylinder, with a radius equal to that of the nanopore $\left(R_{c}=2 \mathrm{~nm}\right.$, where 'c' stands for 'cylinder'), periodic boundary conditions, and an identical adsorption energy (Fig. $2 \mathrm{~A}$ ). Note that in the infinite system, $\delta N_{F}=-\delta N_{B}$ and their respective PSDs are identical. For high frequencies the PSD of the bounded particle number show the same scaling as the one obtained with the nanopore connected to reservoirs, i.e. $1 / f^{\alpha}$ with $\alpha \approx 1.5$. However it exhibits a plateau for frequencies below $0.1 \mathrm{GHz}$ (Fig. $1 \mathrm{C}$, pale blue). The low frequency noise is therefore strongly affected by the presence of the reservoirs, a behavior which we will analyze more in details below.

\section{Modeling of the adsorption kinetics.}

In order to isolate the origin of the pink noise detected in both MD and Brownian simulations, let us focus first on neutral particles reversibly adsorbing at the surface of an effectively infinite cylinder (Fig. 2 A). Our approach extends on the theoretical developments on number fluctuations in a channel by Bezrukov et al. ${ }^{49}$, as well as on the analysis by Levitz et al. on random flights on planar surface ${ }^{50,51}$. We define a state variable $n_{B}^{i}(t)$ which is equal to 1 when a particle is bounded on the surface, and 0 otherwise. The number of bounded particles at any time $t$ follows as $N_{B}(t)=\sum_{i=1}^{N} n_{B}^{i}(t)$. We assume that particles are uncorrelated among each other. The auto-correlation function of the total number of adsorbed particles can be written as a sum

$$
\left\langle N_{B}(0) N_{B}(t)\right\rangle=N^{2} p_{B}^{2}+N p_{B} C_{B B}(t)-N p_{B}^{2},
$$

where $p_{B}$ is the probability for a particle to be adsorbed and $C_{B B}(t)$ the single-ion correlation function, i.e. the probability for an ion to be adsorbed at $t$ given that it is adsorbed at $t=0$. The correlation function $C_{B B}$ can be calculated exactly in terms of the successive adsorption-desorption-diffusion processes of the particles. Explicitly this takes the form in the present case:

$$
C_{B B}(t)=\sum_{n=0}^{\infty}\left[\int_{0}^{\infty} d t_{e} Q\left(t_{e}\right) \prod_{m=1}^{n}\left[\int_{0}^{\infty} d t_{m} P\left(t_{m}\right) \int_{0}^{\infty} d t_{m}^{\prime} J_{\mathrm{c}}\left(t_{m}^{\prime}\right)\right] \times \delta\left(t_{e}+\sum_{k=1}^{n}\left(t_{k}+t_{k}^{\prime}\right)-t\right)\right],
$$

where the outer sum counts the number of desorption and re-adsorption events, $P(t)$ is the distribution of the desorption duration, $P(t)=\lambda \exp (-\lambda t)$, and $Q(t)$ is the survival distribution corresponding to the probability for a particle adsorbed in $t=0$ to remain adsorbed at a time $t$, given by

$$
Q(t)=\int_{t}^{\infty} P\left(t^{\prime}\right) \mathrm{d} t^{\prime}
$$

Finally, $J_{\mathrm{c}}(t)$ is the first-return distribution, defined as the probability for a particle desorbing at time $t=0$ to return to the cylinder surface at $t$. The Laplace transform of $C_{B B}(t)$ allows to perform the sum as a geometric series, leading to the simple result

$$
\tilde{C}_{B B}(\omega)=\frac{\tilde{Q}(\omega)}{1-\tilde{P}(\omega) \tilde{J}_{\mathrm{c}}(\omega)},
$$

where $\tilde{P}(\omega)=\lambda(\lambda+\omega)^{-1}$ and $\tilde{Q}(\omega)=(\lambda+\omega)^{-1}$ are respectively the Laplace transforms of $P(t)$ and $Q(t)$, and $\omega=2 \pi f$.

For the cylinder geometry, the first-return distribution $\tilde{J}_{\mathrm{c}}(\omega)$ is calculated by solving the diffusion equation in cylindrical coordinates

$$
\left(\partial_{t}-D \Delta_{r}\right) G_{\mathrm{c}}\left(r, t \mid r_{0}\right)=0,
$$

where $G_{\mathrm{C}}$ is the radially symmetric Green function and $\Delta_{r}=r^{-1} \partial_{r} r \partial_{r} ; \tilde{J}_{\mathrm{c}}(\omega)$ is related to $G_{\mathrm{c}}$ via the relation $\tilde{J}_{\mathrm{c}}(\omega)=-D \partial_{r} \tilde{G}_{\mathrm{c}}\left(R_{n}, \omega \mid r_{0}=R_{n}\right)$.

The diffusion equation is complemented by the initial condition $G_{\mathrm{c}}\left(r, t=0 \mid r_{0}\right)=\delta\left(r-r_{0}\right) / 2 \pi r$, and the boundary condition on the pore surface which writes $-\left.D \partial_{r} G_{\mathrm{c}}\left(r, t \mid r_{0}\right)\right|_{r=R_{\mathrm{c}}}=k G_{\mathrm{c}}\left(R_{\mathrm{c}}, t \mid r_{0}\right)$, where we introduced the effective adsorption rate $k(\mathrm{~m} / \mathrm{s})$. This leads to an expression for the first-return distribution for an infinite tube of radius $R_{\mathrm{c}}$

$$
\tilde{J}_{\mathrm{c}}(\omega)^{-1}=1+\frac{\sqrt{\omega D}}{k} \frac{I_{1}\left(R_{\mathrm{c}} \sqrt{\omega / D}\right)}{I_{0}\left(R_{\mathrm{c}} \sqrt{\omega / D}\right)},
$$

where $I_{0}(x)$ is the modified Bessel functions and $\partial_{x} I_{0}(x)=I_{1}(x)^{52}$. Further details of the derivation are given in the Supplementary Information. The PSD follows as

$$
S(\omega)=\tilde{C}_{B B}(i \omega)+\tilde{C}_{B B}(-i \omega),
$$

with $i$ the imaginary unit, and $\tilde{C}_{B B}$ from Eq. (4). 

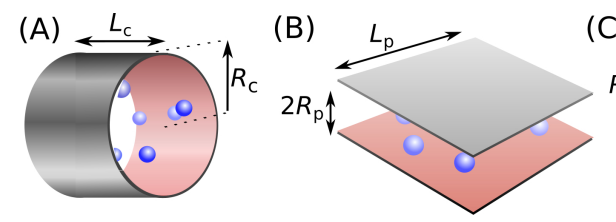

(C)
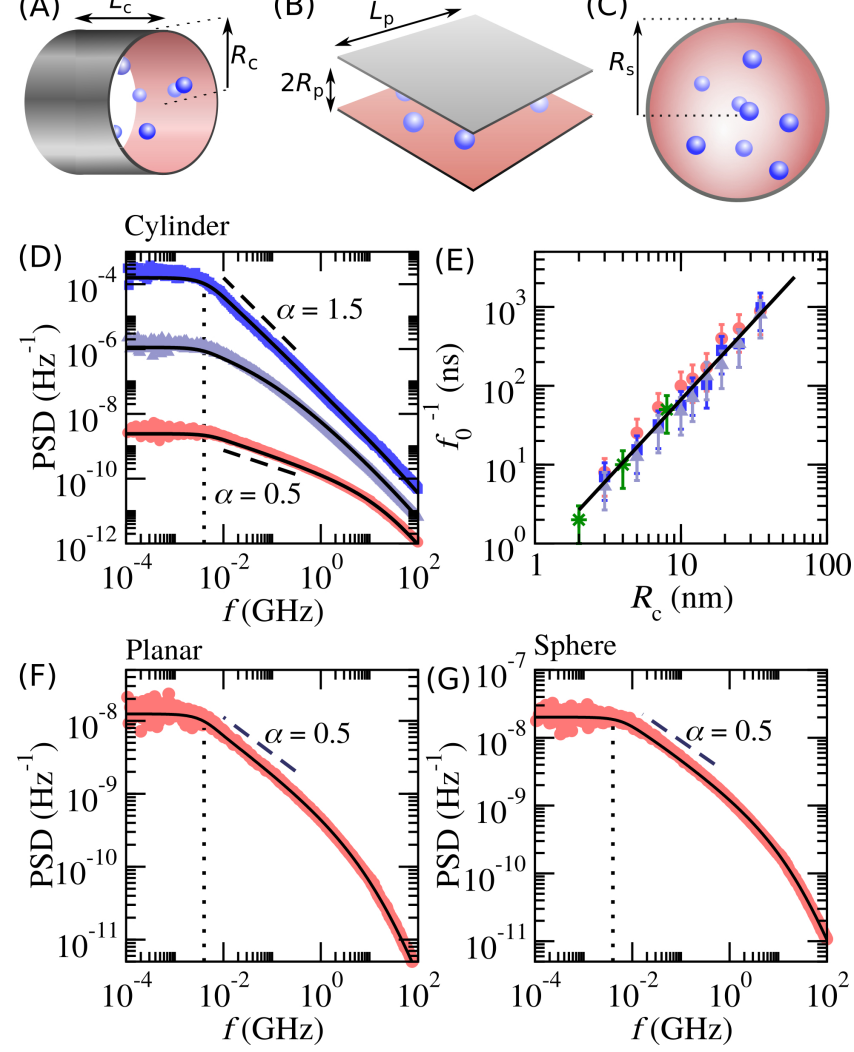

Figure 2: (A) Particles (blue disks) within an effectively infinite cylinder of radius $R_{\mathrm{c}}$ and length $L_{\mathrm{c}}$ with adsorbing boundary (red surface). (B) Particles in between two effectively infinite flat planes of lateral dimension $L_{\mathrm{p}}$ separated by a distance $2 R_{\mathrm{p}}$. (C) Particles within a sphere of radius $R_{\mathrm{s}}$. (D) PSD of the number of bounded particles at the adsorbing surface of an infinite cylinder as function of the frequency $f$ for a tube radius $R_{\mathrm{c}}=20 \mathrm{~nm}$ and a desorption frequency, respectively from bottom to top, $\lambda=100$ (red disks), $\lambda=10$ (gray triangles) and $\lambda=0.01 \mathrm{GHz}$ (blue squares). Dashed lines are guides to the eyes $\propto f^{-\alpha}$, full lines are the analytical calculations (Eq. (7)), and dotted line is $f_{0}$, see text for details. PSDs have been vertically shifted for clarity. (E) Measured inverse cut-off frequency $f_{0}^{-1}$ as function of the cylinder radius $R_{\mathrm{c}}$ for three different values of $\lambda$, the black line is $R_{\mathrm{c}}^{2} / D$ with $D$ the diffusion coefficient of particles. Green stars are neutral MD simulations. (F) PSD for a planar system with separation $2 \times R_{\mathrm{p}}=40 \mathrm{~nm}$ and a desorption frequency $\lambda=100 \mathrm{GHz}$. (G) PSD for a sphere radius $R_{\mathrm{s}}=20 \mathrm{~nm}$ and a desorption frequency $\lambda=100 \mathrm{GHz}$.

We compare this prediction to the results of Brownian simulation in Fig. 2D, using three values of the effective desorption frequency, $\lambda=0.01,10$ and $100 \mathrm{GHz}$ and a radius $R_{\mathrm{c}}=20 \mathrm{~nm}$. Note that the value for the effective adsorption rate $k=22 \mathrm{~m} / \mathrm{s}$ is directly measured from the simulations: $k$ is obtained in terms of the average surface concentration, $c_{\mathrm{s}}\left(\mathrm{mol} \mathrm{m}^{-2}\right)$, and bulk concentration in particles, $c_{\mathrm{b}}\left(\mathrm{mol} \mathrm{m}^{-3}\right)$, according to $k=\lambda c_{\mathrm{s}} / c_{\mathrm{b}}$. Overall, the agreement is shown to be excellent. For a large desorption frequency, $\lambda=100 \mathrm{GHz}$, corresponding to particles quickly returning to diffusing after being adsorbed at the surface, the PSD shows three distinct regimes: a plateau at low frequency $\left(f<4 \cdot 10^{-3} \mathrm{GHz}\right)$, a decrease in $1 / f^{\alpha}$ with $\alpha \approx 1.5$ at high frequency $(f>10 \mathrm{GHz})$, and in between a decrease in $1 / f^{\alpha}$ with $\alpha \approx 0.5$. As the desorption frequency $\lambda$ decreases, the intermediate regime eventually disappears and only two distinct scaling regimes remain (Fig. 2D). Note that a similar impact of the desorption frequency on the scaling of the PSD of confined molecules alternating between diffusion and adsorption has already been reported by Levitz et al. ${ }^{51}$. Regardless of the desorption frequency $\lambda$, the low frequency cut-off $f_{0}$ is given by the diffusive characteristic frequency $f_{0}=f_{D} \approx D / R_{\mathrm{c}}^{2}$, as indicated by both Brownian and neutral MD simulations (Fig. $2 \mathrm{E})$.

The results obtained for cylinders can be generalized to alternative geometries (Fig. 2 B, C), with planar and spherical confinement and adsorbing surfaces. On the theoretical side, for the sphere, the previous framework still holds, with the first-return distribution $\tilde{J}_{\mathrm{c}}(\omega)$ replaced by those calculated for spherical geometry $\tilde{J}_{\mathrm{s}}(\omega)$ (Supplementary Information, Eq. (S45)). For the planar geometry, the equations are slightly more complicated because one must account for particles desorbing from one surface and re-adsorbing to the other (Supplementary Information, Eq. (S59) to Eq. (S86)). As before, the agreement with the simulations is found to be excellent for both the planar geometry of Fig. $2 \mathrm{~B}$, with the particles diffusing in between two adsorbing plates, see Fig. $2 \mathrm{~F}$, and for the spherical geometry of Fig. $2 \mathrm{C}$, see Fig. 2 G.

The study of diffusion of particles within a confined geometry with adsorbing boundaries, either in planar, cylindrical, and spherical, all lead to the same results: the fluctuations of the number of bounded particles exhibits pink noise, with a PSD scaling as $1 / f^{\alpha}$ with $\alpha \in[0.5,1.5]$, for frequencies above a cut-off fixed by the inverse duration of diffusion trajectories $f_{0}=f_{D} \approx D / R_{\mathrm{x}}^{2}$, where $R_{\mathrm{x}}$ is the characteristic dimension of the considered system, i.e. cylinder or sphere radius, or plate separation. The origin of the pink noise is the diffusive return trajectories to the surfaces, combined with the reversible adsorptions. However, the value of the cut-off frequency remains extremely large: indeed even for a large nanopore with radius $R_{n}=100 \mathrm{~nm}, f_{0}=f_{D} \sim 10^{4} \mathrm{~Hz}$ for typical ions. This is in contradiction with experimental results, which exhibit pink noise down to extremely small frequencies in the range of Hz. Furthermore, our previous numerical results for the complete system including reservoirs exhibited pink noise down to frequencies which are far smaller than $f_{D}$, see Fig. $1 \mathrm{CD}$. We thus extend the analysis to explore the effect of reservoirs connecting the pore.

\section{Reservoir effects.}

In order to obtain insight on the cut-off frequency in the presence of reservoirs, we study a simplified geometry 
where an adsorbing system is connected to a single large reservoir. Accordingly we focus on a system made of two concentric spheres of respective radius $R_{\mathrm{cs}}$ and $H_{\mathrm{cs}}$, with $H_{\mathrm{cs}}>R_{\mathrm{cs}}$, as sketched in Fig. $3 \mathrm{~A}$. The surface of the inner sphere is adsorbing, and the surface of the outer sphere is reflecting. Particles are located in between $R_{\mathrm{cs}}$ and $H_{\mathrm{cs}}$. The limit $R_{\mathrm{cs}} \gg H_{\mathrm{cs}}$ is expected to mimic, at least roughly, a nanopore connected to large reservoirs. This geometry captures the elementary process at play when ions escape the nanopores, while keeping analytical calculations tractable.

In order to calculate the analytical expression for the PSD, we solve the diffusion equation in spherical coordinates, $\left(\partial_{t}-D \Delta_{r}\right) G_{\mathrm{S}}\left(r, t \mid r_{0}\right)=0$, where $G_{\mathrm{S}}$ is the radially symmetric Green function and $\Delta_{r}=r^{-2} \partial_{r} r^{2} \partial_{r}$. The initial condition $G_{\mathrm{s}}\left(r, t=0 \mid r_{0}\right)=\delta\left(r-r_{0}\right) / 4 \pi r^{2}$ and the boundary condition on the surface at $r=R_{\mathrm{cs}}$ now writes $\left.\left(k+D \partial_{r}\right) G_{\mathrm{s}}\left(r, t \mid r_{0}\right)\right|_{r=R_{\mathrm{cs}}}=0$. In the limit of large reservoir, $H_{\mathrm{cs}} \gg R_{\mathrm{cs}}$, we obtain the first return distribution of particles onto the inner sphere

$$
\tilde{J}_{\mathrm{cs}}(\omega)^{-1}=1+\frac{D}{k R_{\mathrm{cs}}}+\frac{\sqrt{\omega D}}{k} .
$$

Further details are given in the Supplementary Information. The calculation of the PSD of the number of bounded particles at the inner sphere surface then follows along the same lines as above. The Laplace transform of the correlation function writes

$$
\tilde{C}_{\mathrm{BB}}(\omega)=\frac{\tilde{Q}(\omega)}{1-\tilde{P}(\omega) \tilde{J}_{\mathrm{cs}}(\omega)},
$$

with $\tilde{J}_{\text {cS }}$ from Eq. (8), and the PSD $S(\omega)$ is deduced as $S(\omega)=\tilde{C}_{\mathrm{BB}}(i \omega)+\tilde{C}_{\mathrm{BB}}(-i \omega)$. To assess the validity of the calculations, we compare to numerical simulations in Fig. 3. The inner sphere radius is $R_{\mathrm{cs}}=8 \mathrm{~nm}$, and the outer sphere radius is $H_{\mathrm{cs}}=10 \times R_{\mathrm{cs}}$, which is large enough to ensure that the outer sphere has no impact on the measurement, as confirmed by convergence tests. We performed simulations of uncorrelated Brownian particles. For a large desorption frequency, $\lambda=100 \mathrm{GHz}$, the PSD of the number of bounded particles at the surface of the inner sphere, $N_{B}(t)$, is qualitatively similar to the PSD obtained with confined geometry. The PSD possesses three distinct regimes: a flat PSD at low frequency, a $\mathrm{PSD} \sim f^{-3 / 2}$ at high frequency, and an intermediate regime with $\mathrm{PSD} \sim f^{-1 / 2}$ (Fig. $3 \mathrm{~B}$ ). As the desorption frequency decreases, the intermediate regime disappears. The agreement of the theoretical predictions with the simulation results is excellent, see Fig. 3 B.

But these results are mostly interesting to obtain further insights on the cut-off frequency, which is the question at stake here. As highlighted by the simulations, see Fig. $3 \mathrm{~B}$, a crucial difference between the concentric spheres and confined system is that the low frequency cut-off is pushed down to much lower frequency as
(A)

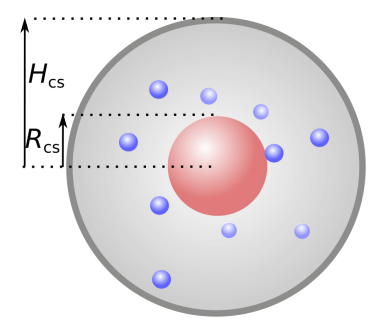

(B)

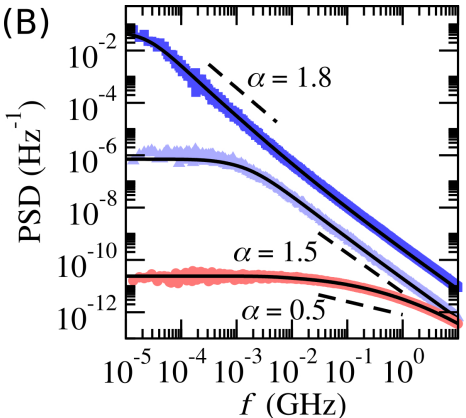

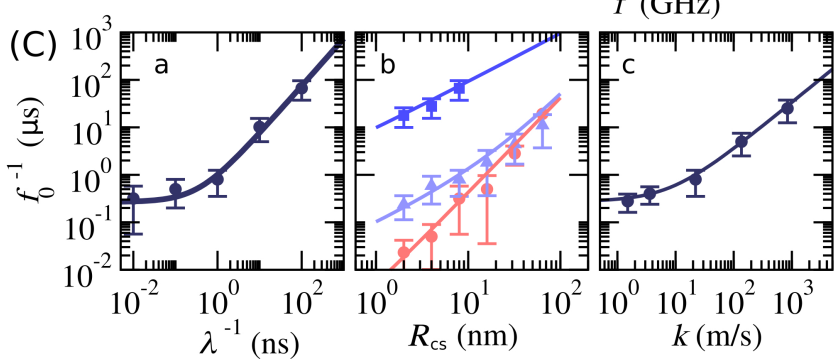

Figure 3: (A) Concentric spheres of respective radius $R_{c s}$ and $H_{c s}$, with adsorbing boundary in red and reflecting boundary in gray. (B) PSD of the number of bounded particles at the adsorbing surface of concentric spheres as function of the frequency $f$ for spheres of respective radius $R_{\mathrm{cs}}=8 \mathrm{~nm}$ and $H_{\mathrm{cs}}=80 \mathrm{~nm}$, and a desorption frequency, respectively from bottom to top, $\lambda=100$ (red disks), $\lambda=1$ (gray triangles) and $\lambda=0.01 \mathrm{GHz}$ (blue squares). Dashed lines are guides to the eyes PSD $\propto f^{-\alpha}$, full lines are the analytical calculations (Eq. (9) with the result (8)). PSDs have been vertically shifted for clarity. (Ca) Inverse of the low frequency cut-off $f_{0}^{-1}$ as measured from simulations for $R_{\mathrm{cs}}=8 \mathrm{~nm}$ (symbols), as a function of the inverse desorption frequency $\lambda^{-1}$. The full line is Eq. (10), with $k=22 \mathrm{~m} / \mathrm{s}$. (Cb) Inverse of the low frequency cut-off $f_{0}$ as function of the inner sphere radius $R_{\mathrm{cs}}$ for $\lambda=100$ (red disks), $\lambda=1$ (gray triangles) and $\lambda=0.01 \mathrm{GHz}$ (blue squares), and for $k=22 \mathrm{~m} / \mathrm{s}$. (Cc) Inverse of the low frequency cut-off $f_{0}$ as function of the effective adsorption boundary $k$ for $R_{\mathrm{cs}}=8 \mathrm{~nm}$ and $\lambda=1 \mathrm{GHz}$.

compared to the diffusive one when the desorption frequency decreases. The diffusive cut-off $f_{D}=D /\left(2 \pi R_{\mathrm{cs}}^{2}\right)$ takes the value $f_{D} \approx 4 \times 10^{-3} \mathrm{GHz}$, but for the lowest desorption frequency $\lambda$, the scaling behavior PSD $\propto f^{\alpha}$ is preserved down to $\sim 10^{-5} \mathrm{GHz}$ (Fig. $3 \mathrm{~B}$ ). This echoes the results obtained with a nanopore connected to large reservoirs in Fig. 1 C D. Physically, our results indicate that the typical duration of the correlations occurring in the system is much longer in a system connected to reservoirs, as compared with a confined geometry.

The dependency of the cut-off frequency $\omega_{0}$ as a function of the desorption frequency $\lambda$, 'pore size' $R$, and effective adsorption coefficient $k$ is shown in Fig. $3 \mathrm{C}$. This is compared to the analytical prediction obtained from the calculation of the PSD above. The predicted low frequency cut-off $f_{0}=\omega_{0} / 2 \pi$ is deduced from

$$
f_{0}^{-1}=f_{D}^{-1}+f_{\lambda}^{-1},
$$


where $f_{D}=D /\left(2 \pi R_{\mathrm{cs}}^{2}\right)$ and

$$
f_{\lambda}=\frac{\lambda / 2 \pi}{\left(1+k R_{\mathrm{cs}} / D\right)},
$$

where the index ' $\lambda$ ' refers to the dependency in the desorption frequency $\lambda$. These predictions do match the simulation results with varying desorption frequency $\lambda$ and varying inner sphere radius $R_{\mathrm{cs}}($ Fig. $3 \mathrm{Ca}, \mathrm{Cb})$. In order to test further the validity of Eq. (10), we also explored the effect of an artificial increase of the effective desorption rate $k=\lambda c_{\mathrm{s}} / c_{\mathrm{b}}$. To do so, we have added a radial force to the particles forcing them to return to the surface of the inner sphere, hence increasing the average fraction of particles adsorbed at the inner sphere surface (Appendix section). For a variation of $k$ from $1.5 \mathrm{~m} / \mathrm{s}$ to $980 \mathrm{~m} / \mathrm{s}$, the agreement between simulation and Eq. (10) is also excellent.

\section{Back to the nanopore.}

These results allow us to discuss more in depth the results for the realistic nanopore in Fig. 1. Indeed, while the above geometry is simplistic, it does capture the elementary process of particle return which is at play in the reservoirs of the realistic nanopore in Fig. 1. The above Eq. (10) predicts a cut-off of $f_{0}=4 \times 10^{-3} \mathrm{GHz}$ for the system in Fig. 1, which agrees well with the measured cut-off. Note however that in Fig. 1, the cut-off is only clearly visible for the Brownian simulations and not from MD due to numerical limitations at low frequencies.

To validate further Eq. (10) when applied to the case of nanopore, we performed MD simulations using a charged nanopore of varying radius $R_{n}=L_{n}$, with $H_{n}=10 R_{n}$. The surface charge is $\Sigma=60 \mathrm{mC} / \mathrm{m}^{2}$ and salt concentration is $c_{s}=20 \mathrm{mM}$. In order to make the cut-off $f_{0}$ detectable given the limitation of the simulation, we use a high desorption frequency $\lambda \approx 100 \mathrm{Ghz}$. The cut-off predicted by Eq. 10 gives an excellent agreement with simulations, using the value of $k=22 \mathrm{~m} / \mathrm{s}$ (Fig. 4 A). MD simulations of neutral systems and Brownian simulations of uncorrelated particles also fall on the same curve. To further confirm the validity of Eq. 10, we also vary the desorption frequency $\lambda$ for a nanopore of radius $R_{n}=1 \mathrm{~nm}$ and find again an excellent agreement between simulations and Eq. (10) (Fig. 4B).

Finally we report the exponent of the PSD for both the number of bounded $N_{B}$ and free $N_{F}$ particles, where the exponent $\alpha$ is defined from $\mathrm{PSD} \sim f^{-\alpha}$. MD results show that $\alpha$ varies strongly with the bulk salt concentration $c_{s}$, in particular the exponent of the PSD of $N_{F}$ ranges from $\alpha \sim 0.15$ to $\sim 1.3$ (Fig. 4 C). The exponent also depends on the desorption frequency $\lambda$ (Fig. 4D), which echoes the results obtained with the two concentric spheres (Fig. 3 B). Finally, we did vary the surface charge from 60 to $360 \mathrm{mC} / \mathrm{m}^{2}$, but did not observe significant variation of the exponent $\alpha$, nor significant variation of the cut-off $f_{0}$ (Supplementary
Information, Fig. S1). However, the measured increase of $k$ was only by a factor 2 in between $\Sigma=60$ and $360 \mathrm{mC} / \mathrm{m}^{2}$, which is expected to impact $f_{0}$ by about $10 \%$ according to Eq. (10), hence smaller than the error bars. Note that we did however observe an increase of the level of noise with increasing surface charge $\Sigma$ (Supplementary Information, Fig. S1), in qualitative agreement with the experimental observations in Ref. 25.
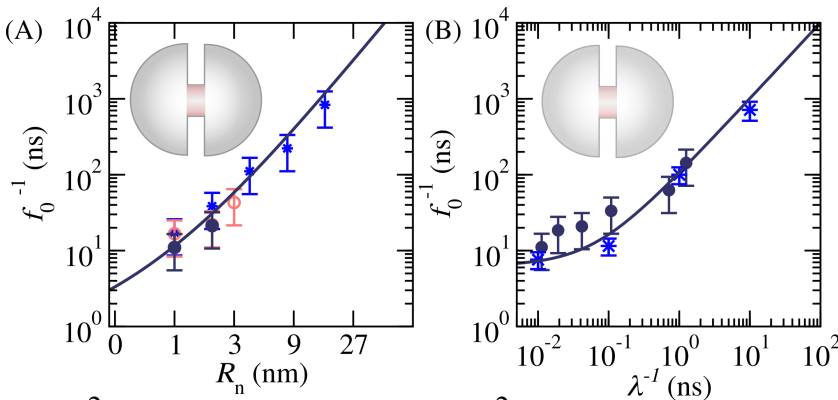

(C)

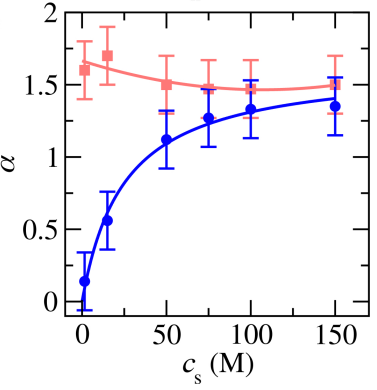

(D)

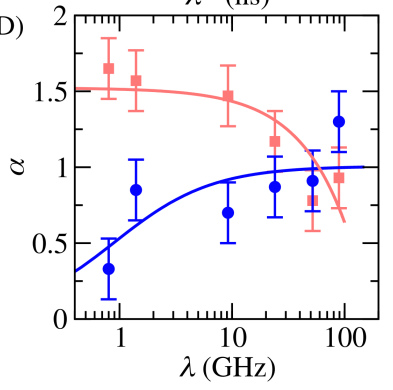

Figure 4: (A) Inverse of the low frequency cut-off $f_{0}$ as function of the nanopore radius $R_{n}$, for a charged nanopore with surface charge $\Sigma=60 \mathrm{mC} / \mathrm{m}^{2}$, salt concentration $c_{s}=20 \mathrm{mM}$, and desorption frequency $\lambda=100 \mathrm{GHz}$ (full symbols). We also report results obtained with a neutral nanopore (open symbols), and using Brownian simulations (stars). The solid line is the prediction in Eq. (10) with $k=22 \mathrm{~m} / \mathrm{s}$. (B) Inverse of the low frequency cut-off $f_{0}$ as a function of the inverse desorption frequency $\lambda^{-1}$ for a nanopore with radius $R_{n}=1 \mathrm{~nm}$. (C) Slope $\alpha$ of the PSD of the number of free particle (blue disks) and number of bounded particle (red squares), for a charged nanopore with surface charge $\Sigma=60 \mathrm{mC} / \mathrm{m}^{2}$, desorption frequency $\lambda=0.8 \mathrm{GHz}$, and nanopore radius $R_{n}=2 \mathrm{~nm}$. Lines are guides to the eye. (D) Slope $\alpha$ as a function of the desorption frequency for a charged nanopore with surface charge $\Sigma=60 \mathrm{mC} / \mathrm{m}^{2}$, nanopore radius $R_{n}=1 \mathrm{~nm}$, and salt concentration $c_{s}=20 \mathrm{mM}$. Lines are guides to the eye.

\section{Discussion.}

In this work we have shown that the fluctuations associated with particles reversibly adsorbing at a solid surface lead to a signal with a PSD scaling as $1 / f^{\alpha}$, with an exponent $\alpha$ typically in the range 0.5 to 1.5 . We recall that the conductance fluctuations are expected to be directly proportional to the PSD of the free ions the fluctuations in the number of free ions, so that the results for the free particles PSD apply to the conductance as well. A crucial remark though is that this low-frequency scaling behavior is observed only above 
a minimum frequency cut-off $f_{0}$. Our predictions show that $f_{0}$ depends not only on the system size and particles diffusion coefficient, but also on the desorption energy and adsorption rate. When the adsorption-desorption process is limiting $\left(k \gg D / R_{\mathrm{c}}\right)$, the cut-off frequency predicted from Eq. (10) is typically

$$
f_{0}=\frac{\omega_{0}}{2 \pi} \approx \frac{\lambda D}{2 \pi k R_{\mathrm{c}}} .
$$

Based on these results, we are now in position to discuss the experimental observations. While the prediction of $1 / f^{\alpha}$ noise is indeed in agreement with the experiments, it is essential to assess that the range of observation for also matehes the experimental ones whether the predicted range for the pink noise also matches experimental ones. In terms of orders of magnitude, simulations showed that a cut-off frequency of about $0.004 \mathrm{GHz}$ is obtained for $\lambda \approx 0.8 \mathrm{GHz}$. While this is still very high compared to the experimental range, one should now estimate the cutoff frequency $f_{0}$ for more realistic values of the adsorption-desorption kinetics. First, the reported values for the experimental desorption frequencies $\lambda$ are rather in the range $\lambda=10^{4}-10^{5} \mathrm{~Hz}$, as measured for the protonization of a transmembrane ionic channels ${ }^{31}$, as well as on the surface of solid-state SiN nanopores ${ }^{10}$. Also based on the association rate constant $k_{R} \sim 10^{11}-10^{12} \mathrm{M}^{-1} \mathrm{~s}^{-1}$ infered for these experiments, one may obtain an estimate for the parameter $k$ (in $\mathrm{m} . \mathrm{s}^{-1}$ ) as $k=k_{R} \times \Gamma$, with $\Gamma$ the number of reactive sites per unit surface $\left(\right.$ in $\mathrm{m}^{-2}$ ). Using the values for $\operatorname{SiN}^{10}\left(\Gamma=0.4 \mathrm{~nm}^{-2}\right)$, one gets $k \sim 100 \mathrm{~m} / \mathrm{s}$. Altogether, for a typical pore of size $R_{\mathrm{n}} \sim 10 \mathrm{~nm}$, Eq.(12) predicts a cut-off frequency as low as $3 \mathrm{~Hz}$ for a solid state nanopore. Similar estimates for nanotubes such as those studied in Ref. 25 lead to an even smaller cutoff in the range of a fraction of Hertz. These estimates show that for the experimentally relevant values of the surface adsorption-desorption coefficients, the cut-off frequency may reach sub-Hertz values and thus lower than the range of frequencies investigated experimentally. In other words the low frequency scaling for the ionic noise $S(f) \sim f^{-\alpha}$ is expected to be observed down to very low frequencies in solid-state nanopore and no white noise regime is observed. However, it is interesting to note that the white-noise regime was actually observed in some measurements performed on protein ion channel ${ }^{31}$, with a pink noise behavior which is observed only above a cut-frequency of about $5 \mathrm{kHz}$. In this system, pink noise is also expected to originate from the reversible adsorption of protons at ionizable sites. Using the reported values for $\lambda$ and $k_{R}$ for this system, one may deduce that $k \sim 10 \mathrm{~m} / \mathrm{s}$. Using a typical radius of $R_{\mathrm{n}} \sim 0.5 \mathrm{~nm}$ for these pores ${ }^{53}$, one gets $f_{0} \sim 1.5 \mathrm{kHz}$ from Eq. (12): this is indeed in the same order of magnitude as the experimentally observed cut-off. These experimental results hence support the importance of surface adsorptiondesorption processes as the origin of low-frequency pink noise. As a further confirmation of surface effects, the amplitude of the noise in carbon nanotubes was measured in Ref. 25 to be strongly dependent on the surface charge. This result is also in agreement with results from the simulation, see supplementary Fig. S1

Altogether our theoretical description demonstrates that the counter-intuitive noise spectrum observed in nanopores takes its origin in the adsorption-desorption process on the pore surface coupled in a crucial way to the long-lasting excursions of the ions into the reservoirs.

\section{Acknowledgments}

LB and RRN acknowledge support from ANR grant Neptune and DFG grant NE 810/11. LB acknowledges support from the European Union's Horizon 2020 Framework Program/European Research Council Advanced Grant 785911 Shadoks. RRN acknowledges support from DFG grant SFB 1078. The authors / SG thank(s) Laurent Joly and Francois Detchevery for valuable discussions.

\section{Appendix}

\section{Molecular dynamics simulations}

We performed Molecular Dynamics (MD) simulations using LAMMPS ${ }^{44}$. The system is a cylindrical nanopore with radius $R_{n}=2 \mathrm{~nm}$ and length $L_{n}=R_{n}$ and connected to large half-spherical reservoirs with radius $H_{n}=20 \mathrm{~nm}$ (Fig. $1 \mathrm{AB}$ ). The solid surfaces are made of a combination of fixed atoms and a solid flat wall that interacts with the liquid by generating a force on the atom in a direction perpendicular to the wall. Water is modeled only in an implicit way, as a medium that has the dielectric permittivity of water $\epsilon_{r}=80$. The interaction between the solute and the water molecules is mimicked by applying a Langevin thermostat on the ions (temperature $T=300 \mathrm{~K}$ ). The same damping time $\tau=23 \mathrm{fs}-$ controlling the amplitude of the viscous drag in the Langevin model - is imposed for both species, to ensure that they have the same diffusion coefficient, with a value $D \approx 1.5 \cdot 10^{-5} \mathrm{~cm}^{2} / \mathrm{s}$, close to experimental ones for typical microions. The equations of motion are integrated using the velocity Verlet algorithm ${ }^{46}$, with a time-step equal to $2 \mathrm{fs}$. The atomic mass of the species is chosen to be $m_{n+}=m_{n-}=39.1 \mathrm{~g} / \mathrm{mol}$, and we consider monovalent ions. A Weeks-Chandler-Andersen potential, keeping only the repulsive part of the Lennard-Jones potential, is used, and all particles (ions and solid atoms) share the same size $\sigma=3 \AA$. Long-range Coulombic interactions are calculated by means of a particle-particle particle-mesh (PPPM) solver.

\section{Brownian particle simulations}

We performed simulation of a random walk using a home-made c++ code. A number $N$ of particle is initially introduced within a finite domain at random position. The domains are either a nanotube connected to half spherical reservoirs (Fig 1 A), an infinite cylinder 
(Fig $2 \mathrm{~A}$ ), or two concentric spheres (Fig $3 \mathrm{~A}$ ). The particles do not interact with each other (particle interaction potentials set equal to 0 ). The simulation time is iterated with constant time step $\Delta t=1 \mathrm{ps}$, and particles positions are iterated following Brownian dynamics (also called overdamped Langevin dynamics) procedure: a Gaussian random number $r$ is generated, and the particle jumps are calculated as $\Delta r=\sqrt{6 D \Delta t} r$, with $D=1.5 \cdot 10^{-5} \mathrm{~cm}^{2} / \mathrm{s}$ the particles diffusion coefficient. When a particle reaches an adsorbing wall, it adsorbs with a probability $p_{\text {abs }}=1$ for a duration $T$ distributed as $P(T)=\lambda \exp (-\lambda T)$ with $\lambda$ the desorption frequency. An adsorbed particle is maintained fix at the surface during $T$. Data acquisition starts after an equilibrium phase. In the case of concentric spheres, if specified, an additional radial force is added to the particles. The additional force is included by adding a deterministic radial velocity to the particles.

\section{References}

(1) Detcheverry, F.; Bocquet, L. Thermal fluctuations in nanofluidic transport. Physical Review Letters 2012, 109, 024501.

(2) Siwy, Z.; Fuliński, A. Origin of $1 / \mathrm{f}^{\alpha}$ Noise in Membrane Channel Currents. Physical Review Letters 2002, 89, 158101.

(3) Chen, P.; Mitsui, T.; Farmer, D. B.; Golovchenko, J.; Gordon, R. G.; Branton, D. Atomic layer deposition to fine-tune the surface properties and diameters of fabricated nanopores. Nano Letters 2004, 4, 1333-1337.

(4) Tabard-Cossa, V.; Trivedi, D.; Wiggin, M.; Jetha, N. N.; Marziali, A. Noise analysis and reduction in solid-state nanopores. Nanotechnology 2007, 18, 305505 .

(5) Tasserit, C.; Koutsioubas, A.; Lairez, D.; Zalczer, G.; Clochard, M. C. Pink noise of ionic conductance through single artificial nanopores revisited. Physical Review Letters 2010, 105, 260602.

(6) Smeets, R. M. M.; Dekker, N. H.; Dekker, C. Noise in solid-state nanopores. Proc. Natl. Acad. Sci. U. S. A. 2008, 105, 095501.

(7) Smeets, R. M. M.; Dekker, N. H.; Dekker, C. Low-frequency noise in solid-state nanopores. Nanotechnology 2009, 20, 095501.

(8) Powell, M. R.; Vlassiouk, I.; Martens, C.; Siwy, Z. S. Nonequilibrium $1 / \mathrm{f}$ noise in rectifying nanopores. Physical Review Letters 2009, 103, 9-12.

(9) Powell, M. R.; Sa, N.; Davenport, M.; Healy, K.; Vlassiouk, I.; Létant, S. E.; Baker, L. a.; Siwy, Z. S.
Noise properties of rectifying nanopores. Journal of Physical Chemistry C 2011, 115, 8775-8783.

(10) Hoogerheide, D. P.; Garaj, S.; Golovchenko, J. A. Probing surface charge fluctuations with solid-state nanopores. Physical Review Letters 2009, 102, 256804 .

(11) Wen, C.; Zeng, S.; Arstila, K.; Sajavaara, T.; Zhu, Y.; Zhang, Z.; Zhang, S.-l. Generalized Noise Study of Solid-State Nanopores at Low Frequencies. 2017,

(12) Wohnsland, F.; Benz, R. 1/f-Noise of open bacterial porin channels. Journal of Membrane Biology 1997, 158, 77-85.

(13) Bezrukov, S. M.; Winterhalter, M. Examining noise sources at the single-molecule level: $1 / \mathrm{f}$ noise of an open maltoporin channel. Physical Review Letters 2000, 85, 202-205.

(14) Howorka, S.; Siwy, Z. Nanopore analytics: sensing of single molecules. Chemical Society reviews 2009, 38, 2360-2384.

(15) He, J.; Liu, H.; Pang, P.; Cao, D.; Lindsay, S. Translocation events in a single-walled carbon nanotube. J. Phys. Condens. Matter 2010, 22.

(16) Merchant, C. A.; Healy, K.; Wanunu, M.; Ray, V.; Peterman, N.; Bartel, J.; Fischbein, M. D.; Venta, K.; Luo, Z.; Johnson, A. T. C.; Drndić, M. DNA translocation through graphene nanopores. Nano Letters 2010, 10, 2915-2921.

(17) Shendure, J.; Ji, H. Next-generation DNA sequencing. Nature Biotechnology 2008, 26, 1135-1145.

(18) Clarke, J.; Wu, H.-C.; Jayasinghe, L.; Patel, A.; Reid, S.; Bayley, H. Continuous base identification for single-molecule nanopore DNA sequencing. Nature Nanotechnology 2009, 4, 265-270.

(19) Reisner, W.; Larsen, N. B.; Silahtaroglu, A.; Kristensen, A.; Tommerup, N.; Tegenfeldt, J. O.; Flyvbjerg, $\mathrm{H}$. Single-molecule denaturation mapping of DNA in nanofluidic channels. Proceedings of the National Academy of Sciences 2010, 107, 1329413299 .

(20) Kowalczyk, S. W.; Hall, A. R.; Dekker, C. Detection of local protein structures along DNA using solid-state nanopores. Nano Letters 2010, 10, 324328.

(21) Lam, E. T.; Hastie, A.; Lin, C.; Ehrlich, D.; Das, S. K.; Austin, M. D.; Deshpande, P.; Cao, H.; Nagarajan, N.; Xiao, M.; Kwok, P.-Y. Genome mapping on nanochannel arrays for structural variation analysis and sequence assembly. Nature Biotechnology 2012, 30, 771-776. 
(22) Garaj, S.; Liu, S.; Golovchenko, J. A.; Branton, D. Molecule-hugging graphene nanopores. 2013, 2013.

(23) Arjmandi-tash, H.; Belyaeva, L. A.; Schneider, F. Single molecule detection with graphene and other two-dimensional materials: nanopores and beyond. Chemical Society Reviews 2015,

(24) Liu, Y.; L., Y. Slowing DNA Translocation in a Nanofluidic Field-Effect Transistor. ACS Nano 2016,

(25) Secchi, E.; Niguès, A.; Jubin, L.; Siria, A.; Bocquet, L. Scaling behavior for ionic transport and its fluctuations in individual carbon nanotubes. Physical review letters 2016, 116, 154501.

(26) Smeets, R. R. M. M.; Keyser, U. U. F.; Krapf, D.; Wu, M. Y. M.; Nynke, H.; Dekker, C.; Dekker, N. H.; Dekker, C. Salt-dependence of ion transport and DNA translocation through solidstate nanopores. Nano Lett. 2006, 6, 89-95.

(27) Zevenbergen, M. A. G.; Singh, P. S.; Goluch, E. D.; Wolfrum, B. L.; Lemay, S. G. Stochastic Sensing of Single Molecules in a Nanofluidic Electrochemical Device. Nano Letters 2011, 2881-2886.

(28) Zevenbergen, M. A.; Singh, P. S.; Goluch, E. D.; Wolfrum, B. L.; Lemay, S. G. Electrochemical correlation spectroscopy in nanofluidic cavities. Analytical chemistry 2009, 81, 8203-8212.

(29) Krause, K.; Mathwig, K.; Wolfrum, B.; Lemay, S. Brownian motion in electrochemical nanodevices. The European Physical Journal Special Topics 2014, 223, 3165-3178.

(30) Singh, P. S.; Lemay, S. G. Stochastic processes in electrochemistry. Analytical chemistry 2016, 88, 5017-5027.

(31) Bezrukov, S. M.; Kasianowicz, J. J. Current noise reveals protonation kinetics and number of ionizable sites in an open protein ion channel. Physical Review Letters 1993, 70, 2352-2355.

(32) Singh, P. S.; Chan, H. S. M.; Kang, S.; Lemay, S. G. Stochastic amperometric fluctuations as a probe for dynamic adsorption in nanofluidic electrochemical systems. Journal of the American Chemical Society 2011, 133, 18289-18295.

(33) Nekolla, S.; Andersen, C.; Benz, R. Noise analysis of ion current through the open and the sugar-induced closed state of the LamB channel of Escherichia coli outer membrane: evaluation of the sugar binding kinetics to the channel interior. Biophysical Journal 1994, 66, 1388-1397.
(34) Andersen, C.; Jordy, M.; Benz, R. Evaluation of the Rate Constants of Sugar Transport Through Maltoporin (LamB) of. J. Gen. Physiol. 1995, 105.

(35) Kullman, L.; Winterhalter, M.; Bezrukov, S. M. Transport of Maltodextrins through Maltoporin: A Single-Channel Study. Biophysical Journal 2002,

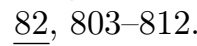

(36) Rostovtseva, T. K.; Bezrukov, S. M. ATP transport through a single mitochondrial channel, VDAC, studied by current fluctuation analysis. Biophysical journal 1998, 74, 2365-73.

(37) Nestorovich, E. M.; Danelon, C.; Winterhalter, M.; Bezrukov, S. M. Designed to penetrate: Timeresolved interaction of single antibiotic molecules with bacterial pores. Proceedings of the National Academy of Sciences 2002, 99, 9789-9794.

(38) Smeets, R. M. M.; Keyser, U. F.; Wu, M. Y.; Dekker, N. H.; Dekker, C. Nanobubbles in solidstate nanopores. Physical Review Letters 2006, 97, $1-4$.

(39) Mathwig, K.; Mampallil, D.; Kang, S.; Lemay, S. G. Electrical cross-correlation spectroscopy: Measuring picoliter-per-minute flows in nanochannels. Physical Review Letters 2012, 109, 118302.

(40) Humphrey, W.; Dalke, A.; Schulten, K. VMD Visual Molecular Dynamics. J. Molec. Graphics 1996, 14, 33-38.

(41) Zorkot, M.; Golestanian, R.; Bonthuis, D. J. The Power Spectrum of Ionic Nanopore Currents: The Role of Ion Correlations. Nano Letters 2016, 16, 2205-2212.

(42) Zorkot, M.; Golestanian, R.; Bonthuis, D. J. Current fluctuations in nanopores: The effects of electrostatic and hydrodynamic interactions. The European Physical Journal Special Topics 2016, 225, 1583-1594.

(43) Hooge, F. N. 1/F Noise Is No Surface Effect. Physics Letters A 1969, 29, 139-140.

(44) Plimpton, S. Fast Parallel Algorithms For Shortrange Molecular-dynamics. J. Comp. Phys. 1995, 117, 1-19.

(45) Schneider, T.; Stoll, E. Molecular-dynamics study of a three-dimensional one-component model for distortive phase transitions. Physical Review B 1978, 17, 1302-1322.

(46) Martys, N.; Mountain, R. Velocity Verlet algorithm for dissipative-particle-dynamics-based models of suspensions. Physical Review E 1999, 59, 37333736 . 
(47) Darden, T.; York, D.; Pedersen, L. Particle mesh Ewald: An $\mathrm{N} \log (\mathrm{N})$ method for Ewald sums in large systems. The Journal of Chemical Physics 1993, 98,10089 .

(48) Papoulis, A.; Pillai, S. U. Probability, Random Variables, and Stochastic Processes, 4th ed.; McGraw Hill: Boston, 2002.

(49) Bezrukov, S. M.; Berezhkovskii, A. M.; Pustovoit, M. A.; Szabo, A. Particle number fluctuations in a membrane channel. The Journal of Chemical Physics 2000, 113, 8206-8211.

(50) Levitz, P. Random flights in confining interfacial systems. Journal of Physics: Condensed Matter 2005, 17, S4059.
(51) Levitz, P.; Bonnaud, P.; Cazade, P.-A.; Pellenq, R.M.; Coasne, B. Molecular intermittent dynamics of interfacial water: probing adsorption and bulk confinement. Soft Matter 2013, $\underline{9}, 8654-8663$.

(52) Wolfram Research, I. Mathematica. 2014; http: //www.wolfram. com/mathematica/.

(53) Menestrina, G. Ionic channels formed by Staphylococcus aureus alpha-toxin: Voltage-dependent inhibition by divalent and trivalent cations. The Journal of Membrane Biology 1986, 90, 177-190. 